\title{
ORIGINAL ARTICLE \\ Medical and psychological factors related to pain in adults with pediatric-onset spinal cord injury: a biopsychosocial model
}

\author{
CB Murray ${ }^{1}, \mathrm{~K}$ Zebracki²,3, KM Chlan ${ }^{4}, \mathrm{AC}^{2} \mathrm{Moss}^{5}$ and LC Vogel ${ }^{6,7}$
}

Study design: A cross-sectional study.

Objectives: The aim of this study is to determine medical and psychological correlates of pain in individuals with pediatric-onset spinal cord injury (SCl).

Setting: Shriners Hospitals for Children-Chicago, Philadelphia and Northern California (USA).

Methods: A total of 187 adults who had sustained an injury before 19 years of age completed interviews that included medical information, standardized measures of psychological functioning (Beck Anxiety Inventory and Patient Health Questionnaire) and a comprehensive pain questionnaire to assess the location, frequency, intensity and duration of pain and distress and disability related to pain.

Results: The findings identified the medical and psychological correlates of pain. Greater symptoms of depression and anxiety were strong and consistent predictors of several aspects of pain, above and beyond the impact of gender, injury-related characteristics and secondary medical complications.

Discussion: The findings support a biopsychosocial model of the development and persistence of pain in individuals with pediatriconset SCl. Interdisciplinary rehabilitation may incorporate psychological treatment such as cognitive-behavioral therapy to reduce the pain and improve functioning. The assessment and treatment of pain in pediatric-onset $\mathrm{SCl}$ is a clinical and research priority.

Sponsorship: This study is supported by funding from Shriners Hospitals for Children and the Craig H. Neilsen Foundation, grant \#324671.

Spinal Cord (2017) 55, 405-410; doi:10.1038/sc.2016.137; published online 27 September 2016

\section{INTRODUCTION}

Pain is a common and debilitating complication in individuals with spinal cord injury (SCI), amplifying the physical impairments by further limiting a person's ability to participate in daily activities and leading to significant decrements in overall health and well-being. ${ }^{1-3}$ Although prevalence rates vary considerably, approximately one-half to two-thirds of the SCI population experience pain. ${ }^{4}$ Because of many contributing biological and psychological factors, pain in SCI may be very difficult to assess and treat. A better understanding of the factors associated with pain provides an empirical foundation to inform clinical decision-making and the development and evaluation of effective pain treatment in individuals with SCI.

According to the biopsychosocial model of pain, the development and persistence of pain after SCI may involve a number of medical and psychological factors. ${ }^{5}$ Medical influences may include pathophysiological factors such as injury-related characteristics (for example, injury level) and secondary complications (for example, urinary tract infections, pressure ulcers). Recent meta-analytic efforts incorporating
82 studies on individuals with adult-onset SCI found that longer duration of injury was associated with increased pain prevalence. ${ }^{4}$ This meta-regression model included several other SCI-related characteristics, such as age of onset, injury completeness and injury level; yet these characteristics were unrelated to pain. ${ }^{4}$ Inconsistencies in reports of injury-related characteristics associated with pain may be due to substantial between-study heterogeneity in the definition and assessment of pain; few studies have included multidimensional pain assessments that consider the full spectrum of pain characteristics, such as pain-related distress and disability in addition to the intensity and frequency of pain. Further, there is a paucity of research that has sought to understand whether SCI-related medical complications may exacerbate painful symptoms in SCI populations. For example, individuals with SCI may experience multiple types of pain (that is, neuropathic as well as nociceptive) secondary to medical complications such as urinary tract infections and pressure ulcers. Thus, further research is needed to examine medical characteristics in relation to multiple aspects of pain.

${ }^{1}$ Department of Psychology, Loyola University, Chicago, IL, USA; ${ }^{2}$ Department of Psychology, Shriners Hospitals for Children, Chicago, IL, USA; ${ }^{3}$ Department of Psychiatry and Behavioral Sciences, Northwestern University Feinberg School of Medicine, Chicago, IL, USA: ${ }^{4}$ Department of Clinical Research, Shriners Hospitals for Children, Chicago, IL, USA; ${ }^{5}$ Department of Psychology, Marquette University, Milwaukee, WI, USA; ${ }^{6}$ Department of Pediatrics, Shriners Hospitals for Children, Chicago, IL, USA and ${ }^{7}$ Department of Pediatrics, Rush Medical College, Chicago, IL, USA

Correspondence: Dr K Zebracki, Department of Psychology, Shriners Hospitals for Children, 2211 N Oak Park Avenue, Chicago, IL 60707, USA.

E-mail: kzebracki@shrinenet.org

Received 17 March 2016; revised 26 July 2016; accepted 22 August 2016; published online 27 September 2016 
Although several studies investigating medical correlates of pain have shown equivocal results, there are strong and consistent data to suggest that psychological factors have a significant and profound impact on the experience, maintenance and aggravation of pain. Longitudinal research utilizing adult-onset SCI populations have pointed to the bidirectional nature of psychological dysfunction and pain, such that negative mood may increase the intensity and frequency of pain, and vice versa. ${ }^{6}$ Therefore, psychological factors should be considered an essential component in research investigations aimed to understand the underlying mechanisms that cause or perpetuate pain in individuals with SCI.

Research has begun to describe key biopsychosocial correlates of pain in adult-onset SCI; yet there are limited data examining these relationships in individuals with pediatric-onset SCI. One study published by our investigative team examined injury-level differences in pain location among adults with pediatric-onset $\mathrm{SCI},{ }^{7}$ although results were limited to describing hand, wrist and shoulder pain. Similar to adult-onset studies, we have also found that anxiety and depression are associated with increased risk for pain that interferes with daily activities in pediatric-onset SCI. ${ }^{8}$ However, research has not tested the predictive utility of a biopsychosocial model of multidimensional aspects of pain in this population. Expanding research in this area is critical because factors associated with pain in individuals with pediatric-onset SCI may be distinguishable from that of adultonset SCI patients. Individuals with pediatric-onset SCI may be at an increased risk for recurrent or persistent pain owing to the long-term experience of injury-related complications (for example, pressure ulcers), multiple surgical interventions, overuse syndromes and skeletal changes secondary to injury. Youth with chronic illnesses also face the added task of managing their condition while navigating challenging biological, psychological and social role changes associated with transitioning through salient developmental periods. ${ }^{9}$ As youth navigate through adolescence (coined 'the storm and stress' period ${ }^{10}$ ), the confluence of developmental factors in the context of adjustment to and management of a chronic illness and disability may exacerbate distress and psychological maladjustment, potentially leading to more severe and persistent pain into adulthood.

The goal of the current study was to identify salient biological and psychological correlates of pain in a sample of adults with pediatriconset SCI, organized within a condition-specific, biopsychosocial model of pain. Although medical factors (beyond the duration of injury) have generally been unrelated to pain in individuals with adult-onset SCI, a comprehensive biopsychosocial model of pain allows for the investigation of medical correlates of pain that are unique to pediatric-onset SCI. As found in our previous research, ${ }^{7}$ we hypothesized that a higher percentage of adults with pediatric-onset paraplegia would experience wrist pain (vs individuals with tetraplegia) as the result of chronic overuse of these muscles from transfers and manual wheelchair use beginning at a young age. In addition, we expected that older pediatric age of injury (that is, adolescence) would predict greater pain and disability. Finally, it was expected that psychological dysfunction (that is, symptoms of anxiety and depression) would impact all dimensions of pain, above and beyond the impact of medical factors.

\section{MATERIALS AND METHODS}

\section{Participants and procedure}

This study was approved by the Institutional Review Board, and consent was obtained before participation. The participants of the current study included a subsample from a larger longitudinal study on long-term psychosocial outcomes in adults with pediatric-onset SCI. ${ }^{11,12}$ The pain questionnaire was not part of the original study protocol and was added at a later time point. All individuals received care at one of the three pediatric SCI programs at Shriners Hospitals for Children: Chicago, Philadelphia and Northern California. Eligible participants were individuals who had sustained an SCI before age 19, were at least 19 years of age at the time of interview, spoke English and did not have a significant brain injury. The data were collected through structured telephone or in-person interviews conducted by trained research assistants. In-person interviews were conducted for participants aged 19-21 years in conjunction with outpatient clinic appointments at their corresponding pediatric Shriners Hospital; all individuals aged 22 and older completed a phone interview. Of the 1143 individuals eligible for participation in the larger longitudinal study, $105(9.2 \%)$ could not be contacted, $95(8.3 \%)$ declined due to lack of interest, $53(4.6 \%)$ died and $409(35.8 \%)$ are still being contacted due to the rolling nature of enrollment, resulting in a sample size of 481 (42.1\%). Of the 481 participants completing interviews between 1996 and 2014, 187 completed the pain questionnaire owing to its later introduction into the study protocol in 2009. The participants who completed the pain questionnaire were more likely to be older at the time of their interview $(M=34.3$ years, s.d. $=7.4)$ and to have a longer injury duration $(M=20.2$ years, s.d. $=8.5)$ compared with individuals who were interviewed before 2009, when the pain questionnaire was not yet added to study measures (age: $M=30.9$ years, s.d. $=5.1, t[479]=5.58$, $P<0.001$; injury duration: $M=20.2$ years, s.d. $=8.5, t[479]=5.36, P<0.001$ ). The two groups did not differ on gender, race, age of injury, or level or severity of injury.

\section{Instruments}

Demographic characteristics. A structured questionnaire designed for this study was used to collect demographic information including age, gender, ethnicity, employment status, education level, marital or dating/relationship status, and living situation.

Medical history. Medical information included injury-related characteristics and complications. Injury-related characteristics (age, level, etiology and severity of injury) were obtained from medical charts and the hospital system's SCI database. The severity of injury was categorized on the basis of the American Spinal Injury Association Impairment Scale utilizing the International Standards for Neurological Classification of Spinal Cord Injury. ${ }^{13}$ Injury-related complications were measured using patient self-report of the presence of muscle spasms (yes/no), pressure ulcers (yes/no ) and total number of urinary tract infections (UTIs) in the previous year.

Psychological functioning. The measures of psychological functioning included patient self-report of depression and anxiety symptoms. The Patient Health Questionnaire- $9^{14}$ was used to assess depressive symptoms in the participants. The symptoms of anxiety were measured using the Beck Anxiety Inventory, ${ }^{15}$ which is a 21 -item measure of subjective, somatic or pain-related symptoms of anxiety and provides an overall total score for anxiety symptoms. The participants indicated how much they were bothered by a particular symptom using a four-point scale $(0=$ not at all to $3=$ severely). Internal consistency for the Patient Health Questionnaire-9 $(\alpha=0.80)$ and Beck Anxiety Inventory $(\alpha=0.75)$ were acceptable.

Pain. A multidimensional pain questionnaire examined the pain location, intensity, frequency, duration, distress and interference, as well as techniques to alleviate pain. The participants indicated all areas of the body where they experienced pain during the past year from a list of standardized body locations identified by Lester, Lefebvre and Keefe. ${ }^{16}$ Usual pain intensity was assessed using an 11 -point numerical rating scale $(0=$ no pain to $10=$ very intense pain). ${ }^{17}$ The pain frequency was rated on a six-point scale $(1=$ less than once per month to $6=$ daily $)^{18}$ and pain duration was also rated on a six-point scale ( $1=\mathrm{a}$ few minutes to $6=$ several days/nights). ${ }^{18}$ Distress due to pain was assessed using a scale from $\mathrm{McGrath}^{19}$ with a five-point scale $(1=$ not at all/no suffering to $5=$ extremely bothered/extreme suffering). The participants also responded to two items related to daily activity and sleep interference owing to pain using a five-point scale ( $1=$ no interference to $5=$ extreme interference). Finally, the participants indicated all techniques used to alleviate pain from a list 
of several options. This multidimensional pain questionnaire has also demonstrated test-retest reliability. ${ }^{20}$

\section{Data analyses}

All analyses were conducted using the Statistical Package for the Social Sciences (SPSS, Armonk, NY, USA), v22. ${ }^{21}$ Preliminary analyses included descriptive statistics to summarize the psychological and pain characteristics of the study sample. To address the primary aim of the current study, Pearson's chi-squared test was first conducted to examine injury-level differences (that is, paraplegia vs tetraplegia) in pain location. To examine the unique contribution of psychological functioning predicting continuous pain outcomes (intensity, frequency, duration, and distress and interference due to pain), hierarchical multiple linear regressions were conducted, controlling first for gender and medical characteristics in the first three steps. Gender was included as a covariate in these analyses because females may be at a greater risk for experiencing more frequent and severe pain compared with males. ${ }^{22}$ Thus, independent variables were entered in the following order: (Step 1) gender; (Step 2) injury-related medical characteristics including injury type (paraplegia vs tetraplegia), age of injury and injury duration; (Step 3) secondary medical complications including muscle spasms, pressure ulcers and UTIs; and (Step 4) psychological characteristics including depression and anxiety. Variables at Step 4 were entered in a forward selection fashion, such that the psychological variable that accounted for the greatest amount of variance in pain (after controlling for demographic and medical characteristics) would be entered first.

\section{Statement of ethics}

The authors certify that all applicable institutional and governmental regulations concerning the ethical use of human volunteers were followed during the course of this research.

\section{RESULTS}

\section{Descriptive data}

Demographic and medical characteristics. The demographic and injury-related characteristics are summarized in Table 1 . In the previous year, one-third (32.6\%) of the participants experienced muscle spasms, and one-half $(51.3 \%)$ of the participants had pressure ulcers. The participants experienced an average of $2.9($ range $=0$ to 24$)$ UTIs in the previous year.

Pain and psychological characteristics. Descriptive statistics for pain characteristics, depression and anxiety are summarized in Table 2. With regard to techniques used to alleviate pain, a small percentage of individuals $(15.0 \%)$ indicated that nothing alleviated their pain. The most common pain management strategy included pharmacological methods; almost half of the sample endorsed benefits of over-thecounter pain mediation (45.6\%) and around one-third indicated the use of prescription pain medication (34.6\%). Non-pharmacological methods for pain management included rest/sleep (27.2\%) and stretching (25.7\%), massage $(16.9 \%)$ and heat application (11.0\%). Less commonly endorsed non-pharmacological strategies included changing positions/exercise (9.6\%), psychological/cognitive-behavioral strategies (that is, deep-breathing, distraction; 9.6\%), acupuncture (2.2\%) and yoga (2.2\%).

Descriptive statistics were used to determine the frequency of scores in the mild and moderate-to-severe symptom ranges for depression and anxiety (Table 2). The participants endorsed low levels of depression and anxiety on average. Moderate to severe levels of depression were reported in $9.1 \%$ of individuals (3.2\% endorsed suicidal thoughts). Moderate to severe anxiety was reported in $8.0 \%$ of individuals. Further, $28.3 \%$ of individuals endorsed current or previous treatment or diagnosis of depression (5.9\% for suicidal thoughts, $3.2 \%$ for suicidal attempt), with slightly lower rates found for anxiety $(22.5 \%)$. A small percentage of the sample (9.6\%) reported receiving psychological treatment for mental health issues in the past year.

\section{Medical and psychological factors related to pain}

First, chi-squared analyses were conducted to determine the relation between injury level (paraplegia vs tetraplegia) and pain location. Results indicated significant group differences in pain location in the sample. Specifically, a significantly higher percentage of participants with tetraplegia endorsed pain in their head or neck $(49.5 \%)$ compared with individuals with paraplegia $\left(25.6 \% ; \chi^{2}[1]=10.68\right.$, $P<0.01)$. In addition, a higher percentage of individuals with paraplegia endorsed pain in their hand/wrist (32.9\% paraplegia, 9.5\% tetraplegia; $\left.\chi^{2}[1]=15.89, P<0.01\right)$ and back $(67.1 \%$ paraplegia, $41.9 \%$ tetraplegia; $\left.\chi^{2}[1]=11.71, P<0.01\right)$ compared with those with tetraplegia. There were no group differences in the following pain locations: chest/abdomen (paraplegia: $11.0 \%$; tetraplegia: $16.2 \%$; $\left.\chi^{2}[1]=1.05, P=0.31\right)$, shoulder $\left(54.2\right.$ vs $59.1 \% ; \chi^{2}[1]=0.39$, $P=0.53)$, arm/elbow (23.2 vs $\left.22.1 \% ; \chi^{2}[1]=0.73, P=0.39\right)$ and lower extremities (36.6 vs $38.1 \% ; \chi^{2}[1]=0.05, P=0.83$ ).

Next, linear regression analyses tested associations among medical and psychological factors and continuous pain variables (Table 3). The multivariate linear models accounted for a significant amount of the variance in usual pain intensity $\left(24.0 \%, \mathrm{~F}_{9,185}=6.18, P<0.001\right)$, pain frequency $\left(16.6 \%, \mathrm{~F}_{9,186}=3.91, P<0.001\right)$, pain duration $(16.5 \%$, $\left.\mathrm{F}_{9,159}=3.29, P<0.01\right)$, distress due to pain $\left(27.6 \%, \mathrm{~F}_{9,159}=6.35\right.$, $P<0.001)$, interference in daily activities $\left(26.3 \%, \mathrm{~F}_{9,159}=5.96\right.$, $P<0.001)$ and interference in sleep $\left(23.9 \%, \mathrm{~F}_{9,159}=5.23, P<0.001\right)$.

When added to the first step, gender was not a significant predictor of any pain characteristic. When injury-related variables were added to the second step, they predicted $5.0 \%$ of the variance in pain intensity and frequency $(P<0.05), 12 \%(P<0.001)$ of the variance in pain duration and $10 \%(P=0.001)$ of the variance in sleep interference due to pain. Tetraplegia was significantly associated with greater sleep interference due to pain. In addition, longer duration of injury and older age at injury predicted greater pain intensity, frequency and interference in sleep owing to pain. Longer duration of injury was also significantly associated with longer pain duration. When secondary condition variables were added to the third step, they predicted an additional $9.0 \%$ of the variance in pain intensity $(P=0.001), 6.0 \%$ $(P=0.01)$ of the variance in pain frequency and $10 \%(P=0.001)$ of the variance in daily interference due to pain. A greater number of UTIs were associated with greater pain intensity and daily activity interference. The presence of muscle spasms in the previous year predicted more intense pain.

Finally, we examined the unique contribution of psychological maladjustment predicting pain characteristics, above and beyond the effect of demographic and medical factors. Overall, psychological maladjustment was significantly associated with the majority of pain characteristics, accounting for an additional $5.0-18.0 \%$ of variance in pain above and beyond the influence of medical factors such as injury level, age of injury, duration of injury, muscle spasms and UTIs. Specifically, symptoms of depression explained a significant proportion of variance in pain frequency $\left(\Delta R^{2}=0.05\right.$ or $\left.5.0 \%, P=0.001\right)$, pain-related distress $\left(\Delta R^{2}=0.18\right.$ or $\left.18.0 \%, P<0.001\right)$ and sleep interference due to pain $\left(\Delta R^{2}=0.09\right.$ or $\left.9.0 \%, P<0.001\right)$. Likewise, symptoms of anxiety explained a significant proportion of variance in pain intensity $\left(\Delta R^{2}=0.09\right.$ or $\left.9.0 \%, P<0.001\right)$, pain-related distress $\left(\Delta R^{2}=0.04\right.$ or $\left.4.0 \%, P=0.01\right)$ and daily activity interference due to pain $\left(R^{2}=0.11\right.$ or $\left.11.0 \%, P<0.001\right)$ after controlling for gender and medical variables. The symptoms of depression and anxiety were unrelated to pain duration, potentially due to the higher percentage $(12 \%)$ of variance explained by injury-related factors in this model. 
Table 1 Sociodemographic and injury characteristics $(n=187)$

\begin{tabular}{|c|c|c|}
\hline Characteristics & $\mathrm{N}(\%) / \mathrm{M}(\mathrm{s} . d$.$) / median$ & Range \\
\hline \multicolumn{3}{|l|}{ Sociodemographics } \\
\hline Age at interview (years) & $34.3(7.4)$ & $19.0-52.0$ \\
\hline Male & $116(62 \%)$ & \\
\hline \multicolumn{3}{|l|}{ Ethnicity/race } \\
\hline Caucasian & $156(83.4 \%)$ & \\
\hline \multicolumn{3}{|l|}{ Employment } \\
\hline $\begin{array}{l}\text { Employed (full- and } \\
\text { part-time) }\end{array}$ & $89(47.6 \%)$ & \\
\hline Unemployed ${ }^{\mathrm{a}}$ & $78(41.7 \%)$ & \\
\hline Student & $20(10.7 \%)$ & \\
\hline University degree or higher & $88(47.1 \%)$ & \\
\hline \multicolumn{3}{|l|}{ Income (yearly) b } \\
\hline Personal & Median $=\$ 25500$ & $\$ 0-1000000$ \\
\hline Combined/family & Median $=\$ 51000$ & $\$ 1000-1000000$ \\
\hline Married & $57(30.5 \%)$ & \\
\hline \multicolumn{3}{|l|}{ Injury characteristics } \\
\hline Age at injury (years) & 14.1 (4.5) Median = 15.6 & 0-19.0 \\
\hline Time since injury (years) & $20.2(8.5)$ & $1.0-45.1$ \\
\hline \multicolumn{3}{|l|}{ Injury severityc } \\
\hline C1-C4 ABC & $29(15.5 \%)$ & \\
\hline C5-C8 ABC & $65(34.8 \%)$ & \\
\hline T1-S5 ABC & $78(41.7 \%)$ & \\
\hline AIS D & $14(7.5 \%)$ & \\
\hline \multicolumn{3}{|l|}{ Level of injury } \\
\hline Tetraplegia & $105(56.1 \%)$ & \\
\hline Paraplegia & $82(43.9 \%)$ & \\
\hline AIS A (\% complete $)^{d}$ & $133(71.1 \%)$ & \\
\hline \multicolumn{3}{|l|}{ Etiology of injury } \\
\hline Vehicular/pedestrian & $95(50.8 \%)$ & \\
\hline Violence & $12(6.4 \%)$ & \\
\hline Fall/flying objective & $14(7.5 \%)$ & \\
\hline Sports & $44(23.5 \%)$ & \\
\hline Medical/surgical & $20(10.7 \%)$ & \\
\hline Other/unknown & $2(1.1 \%)$ & \\
\hline
\end{tabular}

Abbreviations: AIS, American Spinal Injury Association impairment scale.

alncludes homemakers, retirees and unemployed individuals (activity and not activity looking).

${ }^{\mathrm{b}} \mathrm{N}$ missing for personal yearly income $=15 ; N$ missing for combined family income $=35$.

${ }^{\mathrm{C}} \mathrm{N}$ missing for injury severity $=1$.

${ }^{d}$ AIS (American Spinal Injury Association Impairment Scale) assessed by trained medical professional.

\section{DISCUSSION}

This study is the first to provide information on key biopsychosocial correlates of pain in individuals with pediatric-onset SCI to inform multidimensional pain assessment and management in this unique population. Findings demonstrated that pain is common in pediatriconset SCI. Pain occurred in approximately $86 \%$ of the study sample, and almost half of these individuals reported daily, non-remitting pain. The high occurrence and persistence of pain highlights the magnitude of pain problems in pediatric-onset SCI, which may develop into a lifelong and debilitating complication.

Several medical or pathophysiological factors may be associated with pain. Although there is inconsistent evidence to support the
Table 2 Pain and psychological characteristics $(n=187)$

\begin{tabular}{ll}
\hline Characteristics & $\mathrm{N}(\%) / \mathrm{M}(\mathrm{s.d})$. \\
\hline Pain location & \\
Head/neck & \\
Chest/abdomen & $73(39.0 \%)$ \\
Shoulder & $26(13.9 \%)$ \\
Arm/elbow & $91(48.7 \%)$ \\
Hand/wrist & $38(20.3 \%)$ \\
Lower extremities $^{\text {b }}$ & $37(19.8 \%)$ \\
Back & $70(37.4 \%)$ \\
& $99(52.9 \%)$
\end{tabular}

Pain intensity (VAS 0-10)

3.69 (2.48), range $0-10$

Pain frequency

Less than once per month $\quad 42(22.5 \%)$

$1-3$ times per month $\quad 29(15.5 \%)$

1 time per week $11(5.9 \%)$

2-3 times per week $\quad 17(9.1 \%)$

4-5 times per week $\quad 9(4.8 \%)$

Daily

$79(42.4 \%)$

Pain duration ${ }^{\text {d }}$

A few minutes $\quad 12(7.5 \%)$

$1 \mathrm{~h}$ or less $\quad 18(11.3 \%)$

A few hours $\quad 38(23.8 \%)$

Most of the day $\quad 39(24.4 \%)$

All day and night $16(10.0 \%)$

Several days and nights $\quad 37(23.1 \%)$

Distress due to pain ${ }^{\text {d }}$

Not at all

$56(35.0 \%)$

$62(38.8 \%)$

$26(16.3 \%)$

$\begin{array}{ll}\text { A lot } & 8(5.0 \%) \\ \text { Extreme } & 8(5.0 \%)\end{array}$

Extreme

$69(43.1 \%)$

$46(28.8 \%)$

$26(16.3 \%)$

$10(6.3 \%)$

$9(5.6 \%)$

Extreme

$63(39.4 \%)$

$46(28.8 \%)$

$28(17.5 \%)$

$17(10.6 \%)$

$6(3.8 \%)$

Extreme

3.3 (4.0), range 0-19

$47(25.1 \%)$

$17(9.1 \%)$

Moderate to severe (score $>10$ )

4.3 (7.4), range 0-46 $23(12.3 \%)$ $15(8.0 \%)$

Anxiety symptoms (BAI)

Total score (0-63)

Mild (score 8-15)

Moderate to severe (score $>16$ )

Abbreviations: BAI, Beck Anxiety Inventory: PHQ-9, Patient Health Questionnaire-9. a $N=186$.

bLower extremities: category includes pelvis/hip, butt, leg, knee, ankles and feet.

'Back: category includes spine, lower back and upper back.

${ }^{\mathrm{d}} N=160$ as these questions regarding duration, distress and interference were only administered to participants endorsing pain of some level (greater than 0 ) and frequency in the past year.

Participants reported on pain characteristics over the previous year. 
Table 3 Biopsychosocial predictors of pain

\begin{tabular}{|c|c|c|c|c|c|c|c|c|c|c|c|c|c|}
\hline \multirow[t]{3}{*}{ Step } & \multirow[t]{3}{*}{ Predictors } & \multicolumn{12}{|c|}{ Pain characteristics } \\
\hline & & \multicolumn{2}{|c|}{ Intensity (average) } & \multicolumn{2}{|c|}{ Frequency } & \multicolumn{2}{|c|}{ Duration } & \multicolumn{2}{|c|}{ Distress } & \multicolumn{2}{|c|}{ Daily interference } & \multicolumn{2}{|c|}{ Sleep interference } \\
\hline & & $\beta$ & $\Delta \mathrm{R}^{2}$ & $\beta$ & $\Delta \mathrm{R}^{2}$ & $\beta$ & $\Delta \mathrm{R}^{2}$ & $\beta$ & $\Delta \mathrm{R}^{2}$ & $\beta$ & $\Delta \mathrm{R}^{2}$ & $\beta$ & $\Delta \mathrm{R}^{2}$ \\
\hline 1 & Gender & 0.13 & 0.02 & 0.03 & $<0.01$ & 0.04 & $<0.01$ & 0.04 & $<0.01$ & 0.01 & 0.00 & 0.11 & 0.01 \\
\hline \multirow[t]{3}{*}{2} & Injury level & -0.04 & & 0.05 & & 0.14 & & 0.09 & & -0.07 & & $0.16^{*}$ & \\
\hline & Age of injury & $0.24 * *$ & & $0.23 *$ & & 0.13 & & 0.10 & & $0.20 *$ & & $0.24 * *$ & \\
\hline & Duration of injury & $0.18^{*}$ & 0.05 & $0.16^{*}$ & 0.05 & $0.35^{* * *}$ & 0.12 & 0.16 & 0.03 & $0.22^{*}$ & 0.04 & $0.27^{* *}$ & 0.10 \\
\hline \multirow[t]{3}{*}{3} & Muscle spasms & 0.13 & & $0.15^{*}$ & & 0.14 & & 0.04 & & 0.10 & & 0.09 & \\
\hline & UTIS & $0.23^{* *}$ & & 0.11 & & 0.03 & & 0.15 & & $0.23^{* *}$ & & 0.14 & \\
\hline & Pressure ulcers & 0.05 & 0.09 & 0.11 & 0.06 & 0.07 & 0.03 & 0.03 & 0.03 & 0.13 & 0.10 & -0.01 & 0.03 \\
\hline \multirow[t]{2}{*}{4} & Depression Sx & 0.10 & 0.01 & $0.23^{* *}$ & 0.05 & 0.11 & 0.01 & $0.43^{* * *}$ & 0.18 & 0.14 & 0.01 & $0.30 * * *$ & 0.09 \\
\hline & Anxiety Sx & 0.30 ** & 0.09 & 0.13 & 0.01 & -0.07 & 0.00 & $0.25^{* *}$ & 0.04 & $0.34 * * *$ & 0.11 & 0.11 & 0.00 \\
\hline
\end{tabular}

Gender was coded as $0=$ male and $1=$ female; injury level was coded as $0=$ paraplegia and $1=$ tetraplegia; muscle spasm was coded as $0=$ no and $1=$ yes in the past year; pressure ulcers were coded as $0=$ no and $1=$ yes in the past year; the figure for UTIs (urinary tract infections) shows the total number in the past year. ${ }^{*} P<0.05 ;{ }^{*} P<0.01 ;{ }^{* * *} P<0.001$.

association between injury level and pain in studies on adult-onset SCI, previous work supports the current study's finding that individuals with tetraplegia are more likely to endorse head/neck pain compared with those with paraplegia. ${ }^{23,24}$ Neck pain in individuals with tetraplegia may be caused by neuropathic pain from the initial cervical injury, muscle strain from self-care activities (eating, brushing and facial hygiene) or muscle deconditioning. Similar to previous research published by the same investigative team, ${ }^{7}$ findings also demonstrated that individuals with pediatric-onset paraplegia were more likely to endorse hand/wrist pain. Increased risk for recurrent or persistent pain in the hands and wrists may result from extensive and long-term use of these muscles and joints for activities such as transfers and manual wheelchair propulsion. Consequently, teaching proper body mechanics and monitoring of these activities through techniques such as motion analyses may help promote effective movement and thereby preserve function and minimize pain.

The current study also found that individuals with pediatric-onset tetraplegia experience greater sleep interference due to pain compared with individuals with paraplegia. Although there are limited research data on injury-level differences in sleep interference due to pain in SCI populations, individuals with tetraplegia experience greater below-level neuropathic pain that tends to be severe and refractory to treatment ${ }^{25}$ as well as greater mobility restrictions that prevent shifting to a more comfortable position, potentially leading to greater difficulties with sleep onset and maintenance. Although research has found that persistent and intense pain may be associated with significant disruptions in sleep quality in individuals with $\mathrm{SCI},{ }^{26,27}$ the type or quality of pain experienced by individuals with tetraplegia may be particularly detrimental to sleep. Further, because of the bidirectional nature of sleep and pain, it is also likely that behavioral and physiological sleep disorders that reduce the quality and quantity of sleep exacerbate pain and related psychological (for example, mood) and physical (for example, overweight/obesity) health sequelae. ${ }^{28,29}$ Although outside the scope of the current study, future research may utilize multimodal sleep assessments (for example, questionnaires, polysomnography, actigraphy) to expand the limited data on injurylevel differences in the nature, severity and impact of sleep disturbances in pediatric-onset SCI.

In addition, findings demonstrated that longer duration of injury was significantly related to several dimensions of pain, including greater pain severity, frequency, duration and daily activity and sleep interference due to pain. Additional years of an injury may compound the risk for pain owing to the long-term impact of medical and physical complications such as overuse syndromes and surgical procedures. ${ }^{25}$ Continual monitoring of pain throughout the individual's lifespan may help to prevent and reduce the burden of chronic pain. Moreover, older age at injury onset was associated with multiple pain outcomes; yet there is a paucity of data to support this link in adult-onset SCI. Acquiring an SCI at an older pediatric age may have important implications for the development of chronic pain that persists into adulthood. Adolescents may be particularly vulnerable to the development of pain and disability owing to the challenge of managing a chronic condition in the context of biological and psychosocial changes unique to this period of development (for example, pubertal changes, peer and academic stress). ${ }^{30}$ Although outside the scope of the current study, longitudinal research may investigate whether individuals who acquire an SCI during childhood, preadolescence period, adolescence period or adulthood evidence differential pain trajectories, including the onset, intensity and persistence of pain. Such research may help focus screening and early prevention efforts on individuals who are at risk for the development of chronic pain.

Despite overall low levels of anxiety and depression, psychological dysfunction was associated with multiple aspects of pain in adults with pediatric-onset SCI, above and beyond the impact of medical characteristics. The cross-sectional nature of this study precludes our ability to make conclusive statements on directionality. Nevertheless, the high prevalence of chronic, unremitting pain experienced by individuals in this population as well as current findings showing unique contributions of depression and anxiety on multiple aspects of pain indicates that psychological maladjustment may compound the risk for debilitating pain in this medically vulnerable population. Several systematic reviews have found that cognitive-behavioral psychosocial interventions alleviate pain and disability in children and adults with chronic pain; ${ }^{31,32}$ yet the efficacy of a multidisciplinary cognitive-behavioral intervention for individuals with SCI has not yet been established because of the limited data available. ${ }^{33}$ Effective cognitive-behavioral interventions that integrate strategies to reduce depression and anxiety may be critical to minimize the burden of pain and distress in this population.

Despite our strong and consistent findings on the connection between psychological maladjustment and pain, very few participants endorsed the use of psychological strategies (for example, distraction, deep-breathing) to alleviate pain. Low rates of non-pharmacological 
pain management may reflect the stigma attached to seeking psychological care and current trends toward pharmacology as the first line of pain treatment in this population. In fact, recently published work by our investigative team found that over one-fourth of individuals with pediatric-onset SCI required routine use of opioids and anticonvulsants, potentially to treat severe and unremitting neuropathic or musculoskeletal pain. Unfortunately, several studies have shown that chronic pain after SCI is often refractory to pharmacological treatment, and long-term pain medication may be associated with unwanted side effects such as constipation and increased risk for addiction or abuse. The integration of psychological treatment or a stepped-care approach to pain management may lead to gradual reductions in inappropriate or excessive pain medication use.

The results of the current study should be considered in light of several limitations. All the study participants have previously received care from a single pediatric specialty hospital system and the majority were Caucasian; thus, our study findings may not generalize to individuals with SCI who have received care at different facilities and those of other ethnicities. On the other hand, the three SCI programs have a wide catchment area serving all regions of the United States and individuals of varying socioeconomic status. Although beyond the scope of the current study, future research may assess the impact of psychological diagnoses and treatment before injury onset on the development of chronic pain. Data allowing for the systematic classification of SCI pain (that is, musculoskeletal, visceral, at-level and below-level neuropathic) were not available, limiting the degree to which the results can be compared with past research. The data on pain prescription medication lacked specificity to be included in the analyses as we did not inquire whether certain medications (for example, antidepressants) were prescribed for pain, depression or co-occurrence of these symptoms. Although there is likely to be a bidirectional link between psychological maladjustment and pain, the cross-sectional nature of the current study precluded our ability to test directionality. Longitudinal research will allow for comprehensive evaluations of the psychosocial risk factors of pain within the biopsychosocial model that were outside the scope of the current study, including developmentally salient cognitive (for example, pain beliefs, catastrophizing) and social (peer and family support, spousal and family responses to pain) influences.

Overall, study findings underscore the importance of evaluating the mechanisms of pain in pediatric-onset SCI using a biopsychosocial framework to advance effective, multimodal and condition-specific pain interventions. Continued monitoring of pain throughout the individual's lifespan, preventative treatment efforts, and incorporation of multidimensional pain assessment and psychological pain treatment into tertiary care and interdisciplinary rehabilitation programs will be critical to decrease suffering and promote functioning in individuals with pediatric-onset SCI.

\section{DATA ARCHIVING}

There were no data to deposit.

\section{CONFLICT OF INTEREST}

The authors declare no conflict of interest.

\section{ACKNOWLEDGEMENTS}

The completion of this manuscript was supported by funding from Shriners Hospitals for Children and the Craig H. Neilsen Foundation (grant \#324671). This study is part of an ongoing, longitudinal study on psychosocial outcomes in adults with pediatric-onset spinal cord injury (SCI).
1 Craig A, Tran Y, Middleton J. Psychological morbidity and spinal cord injury: a systematic review. Spinal Cord 2009; 47: 108-114.

2 Jensen MP, Hoffman AJ, Cardenas DD. Chronic pain in individuals with spinal cord injury: a survey and longitudinal study. Spinal Cord 2005; 43: 704-712.

3 Ullrich PM, Lincoln RK, Tackett MJ, Miskevics S, Smith BM, Weaver FM. Pain depression, and health care utilization over time after spinal cord injury. Rehabil Psychol 2013; 58: 158-165.

4 van Gorp S, Kessels AG, Joosten EA, Patijn N. Pain prevalence and its determinants after spinal cord injury: a systematic review. Eur J Pain 2015; 19: 5-14.

5 Widerström-Noga EG, Finnerup NB, Siddall PJ. Biopsychosocial perspective on a mechanisms-based approach to assessment and treatment of pain following spinal cord injury. J Rehabil Res Dev 2009; 46: 1-12.

6 Kennedy P, Hasson L. The relationship between pain and mood following spinal cord injury. J Spinal Cord Med 2016; 1: 1-5.

7 Vogel LC, Chlan KM, Zebracki K, Anderson CJ. Long-term outcomes of adults with pediatric-onset spinal cord injuries as a function of neurological impairment. J Spinal Cord Med 2013; 34: 60-66.

8 January AM, Zebracki K, Chlan KM, Vogel LC. Mental health and risk of secondary medical complications in adults with pediatric-onset spinal cord injury. Top Spinal Cord Inj Rehabil 2014; 20: 1-12.

9 Suris JC, Michaud PA, Viner R. The adolescent with chronic condition. Part 1: development issues. Arch Dis Child 2004; 89: 938-942.

10 Cicchetti D, Rogosch F. A developmental psychopathology perspective on adolescence. J Consult Clin Psychol 2002; 70: 6-20.

11 January A, Zebracki K, Chlan K, Vogel LC. Understanding posttraumatic growth following pediatric-onset spinal cord injury: the critical role of coping strategies for facilitating positive psychological outcomes. Dev Med Child Neurol 2015; 57: 1143-1149.

12 Vogel L, Chlan K, Zebracki K, Anderson CJ. Long-term outcomes of adults with pediatric-onset spinal cord injuries as a function of neurological impairment. J Spinal Cord Med 2011; 34: 60-66.

13 Kirshblum SC, Burns SP, Biering-Sorensen F, Donovan W, Graves DE, Jha A et al. International standards for neurological classification of spinal cord injury. J Spinal Cord Med 2011; 34: 535-546.

14 Spitzer RL, Kroenke K, Williams JBW. Validation and utility of a self-report version of PRIME-MD: the PHQ primary care study. JAMA 1999; 282: 1737-1744.

15 Beck AT, Epstein N, Brown G, Steer RA. An inventory for measuring clinical anxiety: psychometric properties. J Consult Clin Psychol 1988; 56: 893-897.

16 Lester N, Lefebvre JC, Keefe FJ. Pain in young adults-III: relationships of three pain-coping measures to pain and activity interference. Clin J Pain 1996; 12: 291-300.

17 von Baeyer CL. Numerical rating scale for self-report of pain intensity in children and adolescents: recent progress and further questions. Eur J Pain 2009; 13: 1005-1007.

18 Palermo TM, Valenzuela D, Stork P. A randomized trial of electronic versus paper pain diaries in children: Impact on compliance, accuracy, and acceptability. Pain 2004; 107: 213-219.

19 McGrath PJ. Pain in Children: Nature, Assessment, and Treatment. Guilford Press: New York, NY, USA. 1990.

20 Klepper S. Effects of an eight-week physical conditioning program on disease signs and symptoms in children with chronic arthritis. Arthritis Care Res 1999; 12: 52-60.

21 IBM SPSS Statistics for Windows [computer program]. Version 22.0. IBM Corp: Armonk, NY, USA. 2013.

22 Fillingim RB, King CD, Ribeiro-Dasilva MC, Rahim-Williams B, Riley JL 3rd. Sex, gender, and pain: a review of recent clinical and experimental findings. J Pain 2009; 10: $447-485$.

23 Turner JA, Cardenas DD, Warms CA, McClellan CB. Chronic pain associated with spinal cord injuries: a community survey. Arch Phys Med Rehabil 2001; 82: 501-508.

24 Ullrich PM, Jensen MP, Loeser JD, Cardenas DD. Pain intensity, pain interference and characteristics of spinal cord injury. Spinal Cord 2008; 46: 451-455.

25 Siddall PJ, McClelland JM, Rutkowski SB, Cousins MJ. A longitudinal study of the prevalence and characteristics of pain in the first 5 years following spinal cord injury. Pain 2003; 103: 249-257.

26 Budh CN, Hultling C, Lundeberg T. Quality of sleep in individuals with spinal cord injury: a comparison between patients with and without pain. Spinal Cord 2005; 43 : 85-95.

27 January AM, Zebracki K, Chlan KM, Vogel LC. Sleep, well-being, and psychological symptoms in adults with pediatric-onset spinal cord injury. Rehabil Psychol 2015; 60: 328-334.

28 Cappuccio FP, Taggart FM, Kandala N, Currie A, Peile E, Stranges S et al. Meta-analysis of short sleep duration and obesity in children and adults. Sleep 2008; 31: 619-626.

29 Staner L. Comorbidity of insomnia and depression. Sleep Med 2010; 14: 35-46.

30 Feldman SS, Elliott GR. At the Threshold: The Developing Adolescent. Harvard University Press: Cambridge, MA, USA. 1990.

31 Eccleston C, Morley S, Williams A, Yorke L, Mastroyannopoulou K. Systematic review of randomized controlled trials of psychological therapy for chronic pain in children and adolescents, with a subset meta-analysis of pain relief. Pain 2002; 99: 157-165.

32 Williams AC, Eccleston C, Morley S. Psychological therapies for the management of chronic pain (excluding headache) in adults. Cochrane Database Syst Rev 2012; 11 : CD007407.

33 Boldt I, Eriks-Hoogland I, Brinkhof MW, de Bie R, Joggi D, von Elm E. Non-pharmacological interventions for chronic pain in people with spinal cord injury. Cochrane Database Syst Rev 2014; 11: CD009177. 